\title{
Inhomogeneous Problem for the Ginzburg-Landau Equation on Two Dimensional Compact Manifolds
}

\author{
Li Ma and Jing Wang \\ Communicated by Yuxi Zheng, received March 23, 2010.
}

\begin{abstract}
In this paper, we consider an inhomogeneous problem for GinzburgLandau equation on a two dimensional compact manifold with smooth boundary. We prove that the problem has a unique global solution in a suitable space.
\end{abstract}

\section{Contents}

1. Introduction 175

2. Preliminary 177

3. Local existence and blow-up criterion $\quad 179$

4. Global existence 182

References 185

\section{Introduction}

Assume that $M^{d}$ is a compact smooth Riemannian manifold of dimension $d$ with smooth boundary $\partial M$. In this short note, we consider the inhomogeneous initial-boundary problem of the Ginzburg-Landau equation:

$$
\begin{cases}i \partial_{t} u=\Delta u-\lambda\left(|u|^{2}-1\right) u, & x \in M, t \in R \\ u(0, x)=\phi(x) & (x, t) \in \partial M \times R \\ u(t, x)=Q(t, x), & \end{cases}
$$

with the dimension restriction $d=2$, where $\lambda>0$ is a parameter. Here we assume that $Q \in C^{3}(\partial M \times(-\infty, \infty))$ has compact support and satisfies the compatibility condition $\phi(x)=Q(0, x)$ on $\partial M$ in the trace sense.

2000 Mathematics Subject Classification. Primary 35Q55, 35R01.

Key words and phrases. Ginzburg-Landau equation, inhomogeneous problem, global solution.

The research is partially supported by the National Natural Science Foundation of China 10631020 and SRFDP 20060003002.

(c)2010 International Press 
When $M=R^{d}$, the Ginzburg-Landau equation with the parameter $\lambda$ is considered as a physical model for superconductivity and has been studied by many authors. For some interesting results we refer the readers to [5] and [7]. Without loss of generality, we shall fix the parameter $\lambda=1$ and then the equation in (1.1) is similar to the standard Schrodinger equation on manifolds. Though there are a lot of works about the homogeneous problem for schrodinger equation (see, for example, $[\mathbf{1}],[\mathbf{4}],[\mathbf{9}]$ and $[\mathbf{1 0}])$, there are a few about the inhomogeneous problem. New feature appears for inhomogeneous problem. For the inhomogeneous problem, the mass and energy identities are not available, though we have

$$
\partial_{t} \int_{M}|u|^{2}=2 \operatorname{Im} \int_{\partial M}<P, n>\bar{Q},
$$

and

$$
\partial_{t} \int_{M}|\nabla u|^{2}+\frac{1}{2}\left(|u|^{2}-1\right)^{2}=2 R e \int_{\partial M}<P, n>\bar{Q}_{t} .
$$

It seems difficult to control the boundary terms, however in the paper [9], Strauss and $\mathrm{Bu}$ have found interesting identities, which provide a technique of dealing with nontrivial boundary term in the initial-boundary problem for nonlinear schrodinger equation on a smooth domain in $R^{d}$. We notice that the technique is essentially a Pohozaev type identity and it can be extended to the compact manifold with boundary. The new ingredient for us to manage the nonlinear term in (1.1) is to apply the Strichartz estimate on the compact manifold obtained in [2]. We will concentrate our attention to two dimension case, i.e, $d=2$. In this case, we prove the following result.

Theorem 1.1. Let $\phi \in H^{1}(M)$ and $Q \in C^{3}(\partial M \times(-\infty, \infty))$ have compact support and satisfy the compatibility condition $\phi(x)=Q(0, x)$ on $\partial M$ in the trace sense. Then for $(p, q)$ being the Strichartz admissible pair with

$$
\sigma=1-\frac{4}{3 p}>\frac{2}{q}
$$

there exists a unique global solution for the equation (1.1) in the space $C\left(R, H^{1}(M)\right) \cap$ $L_{l o c}^{p}\left(R, W^{\sigma, q}(M)\right)$.

Here, by definition, the Strichartz admissible pair $(p, q)$ satisfies

$$
\frac{2}{p}+\frac{d}{q}=\frac{d}{2},(p, q, d) \neq(2, \infty, 2) .
$$

We remark that our result also holds for the cubic defocusing Schrodinger equation and moreover in this case we have improved the result in $[\mathbf{9}]$. The reason for our restriction to dimension two is because of the Sobolev imbedding theorem in this dimension and generally speaking, the 2-dimensional nonlinear Schrodinger type evolution equation enjoys special structure.

We denote by $n$ the unit outer normal vector field of $\partial M$. In the following, we extend $n$ to be a smooth vector field on $M$ to which we denote by $\xi$. The divergence of $\xi$ is written as $\eta$. We also use the notations:

$$
\begin{gathered}
P:=\left.\nabla u\right|_{\partial M}, \\
f(u)=\left(|u|^{2}-1\right) u,
\end{gathered}
$$




$$
F(u)=\frac{1}{2}\left(|u|^{2}-1\right)^{2} .
$$

$<,>$ denotes the inner product of the Riemannian manifold $M$.

The paper is organized as follows. First we derive in section 2 the identities corresponding mass, energy and the boundary term which will be used later; Second we prove the local existence and blow up criterion by Strichartz estimate in section 3 . In the last part, by showing the $H^{1}$ norm will not blow up at finite time, we get the global result in section 4 .

\section{Preliminary}

In this section, we mainly prove some identities. Two of them correspond to mass conversation and energy conversation in the homogeneous problem and the others are used to control the boundary term. The proof is direct, though for self-contained we give the details here.

Lemma 2.1. Let $u$ be a smooth solution to the equation (1.1), then the following identities hold. First,

$$
\partial_{t} \int_{M}|u|^{2}=2 \operatorname{Im} \int_{\partial M}<P, n>\bar{Q},
$$

Second,

$$
\partial_{t} \int_{M}|\nabla u|^{2}+F(u)=2 R e \int_{\partial M}<P, n>\bar{Q}_{t}
$$

Third,

$$
\begin{aligned}
& \partial_{t} \int_{M} u<\xi, \nabla \bar{u}>-\int_{\partial M} Q \bar{Q}_{t}+\int_{M} \eta u \bar{u}_{t} \\
= & i \int_{M}<\nabla \xi, \nabla u \otimes \nabla \bar{u}>-i \int_{\partial M}|<P, n>|^{2} \\
- & \frac{i}{2} \int_{M} \eta\left(|u|^{2}-1\right)^{2}+\frac{i}{2} \int_{\partial M}\left(|Q|^{2}-1\right)^{2},
\end{aligned}
$$

Fourth,

$$
\begin{aligned}
& \int_{M} i \eta u_{t} \bar{u}=-\int_{M} \eta|\nabla u|^{2} \\
- & \int_{M} \bar{u}<\nabla u, \nabla \eta> \\
+ & \int_{\partial M} \eta \bar{Q}<P, n>+\int_{M} \eta\left(|u|^{2}-1\right)|u|^{2} .
\end{aligned}
$$


Proof. To prove (2.1), we differentiate $|u|^{2}$ in $t$ and then integrate over $M$ to obtain

$$
\begin{aligned}
\partial_{t} \int_{M}|u|^{2} & =\int_{M} u_{t} \bar{u}+u \bar{u}_{t} \\
& =i \int_{M}(\Delta \bar{u} u-\Delta u \bar{u}) \\
& =i \int_{\partial M}<\bar{P}, n>u-<P, n>\bar{u} \\
& =2 \operatorname{Im} \int_{\partial M}<P, n>\bar{Q},
\end{aligned}
$$

where in the second equality we used the equation (1.1).

For (2.2), direct computation shows

$$
\partial_{t} \int_{M}|\nabla u|^{2}=\int_{M}<\nabla u_{t}, \nabla \bar{u}>+<\nabla u, \nabla \bar{u}_{t}>.
$$

Integrating by part, we get

$$
\begin{aligned}
\partial_{t} \int_{M}|\nabla u|^{2}= & -\int_{M} u_{t} \Delta \bar{u}+\int_{\partial M} u_{t}<\bar{P}, n> \\
& -\int_{M} \Delta u \bar{u}_{t}+\int_{\partial M} \bar{u}_{t}<P, n>.
\end{aligned}
$$

Similarly we get

$$
\partial_{t} \int_{M} F(u)=\int_{M}\left(|u|^{2}-1\right)\left(u_{t} \bar{u}+u \bar{u}_{t}\right) .
$$

Combining the identities $(2.5),(2.6)$ with the equation (1.1), we obtain

$$
\begin{aligned}
\partial_{t} \int_{M}|\nabla u|^{2}+F(u) & =\int_{M} u_{t}\left[\left(|u|^{2}-1\right) \bar{u}-\Delta \bar{u}\right]+\int_{M} \bar{u}_{t}\left[\left(|u|^{2}-1\right) u-\Delta u\right] \\
& +\int_{\partial M} Q_{t}<\bar{P}, n>+\int_{\partial M} \bar{Q}_{t}<P, n> \\
& =2 \operatorname{Re} \int_{\partial M} \bar{Q}_{t}<P, n>
\end{aligned}
$$

In order to establish (2.3), denoting the left hand side of (2.3) by $L H S$, we have $(2.7) L H S=\int_{M} u_{t}<\xi, \nabla \bar{u}>+\int_{M} u<\xi, \nabla \bar{u}_{t}>-\int_{\partial M} Q \bar{Q}_{t}+\int_{M} \eta u \bar{u}_{t}$.

Integrating by part we obtain

$$
\begin{aligned}
\int_{M} u<\xi, \nabla \bar{u}_{t}> & =\int_{M}<u \xi, \nabla \bar{u}_{t}>=-\int_{M} \operatorname{div}(u \xi) \bar{u}_{t}+\int_{\partial M} Q \bar{Q}_{t} \\
& =-\int_{M}<\nabla u, \xi>\bar{u}_{t}-\int_{M} \eta u \bar{u}_{t}+\int_{M} Q \bar{Q}_{t} .
\end{aligned}
$$

Substitute (2.8) into the identity (2.7), then

$$
L H S=\int_{M} u_{t}<\xi, \nabla \bar{u}>-\bar{u}_{t}<\xi, \nabla u>.
$$


By the equation, we have

$$
\begin{aligned}
u_{t} \nabla \bar{u}-\nabla u \bar{u}_{t} & =\left(-i \Delta u+i\left(|u|^{2}-1\right) u\right) \nabla \bar{u}-\nabla u\left(i \Delta u-i\left(|u|^{2}-1\right) \bar{u}\right) \\
& =-i(\Delta u \nabla \bar{u}+\nabla u \Delta u)+i\left(|u|^{2}-1\right) \nabla|u|^{2} \\
& =-i d i v \nabla u \otimes \nabla \bar{u}+\frac{i}{2} \nabla\left(|u|^{2}-1\right)^{2} .
\end{aligned}
$$

So it is clear that

$$
\begin{aligned}
L H S & \left.\left.=-i \int_{M}<\xi, \operatorname{div} \nabla u \otimes \nabla \bar{u}\right\rangle+\frac{i}{2} \int_{M}<\xi, \nabla\left(|u|^{2}-1\right)^{2}\right\rangle \\
& =i \int_{M}<\nabla \xi, \nabla u \otimes \nabla \bar{u}>-i \int_{\partial M}|<P, n>|^{2} \\
& -\frac{i}{2} \int_{M} \eta\left(|u|^{2}-1\right)^{2}-\frac{i}{2} \int_{\partial M}\left(|Q|^{2}-1\right)^{2} .
\end{aligned}
$$

The last identity (2.4) is obtained by multiplying the equation by $\eta \bar{u}$

$$
\begin{aligned}
\int_{M} i \eta u_{t} \bar{u} & =\int_{M} \eta \Delta u \bar{u}+\int_{M} \eta\left(|u|^{2}-1\right)|u|^{2} \\
& =-\int_{M}<\nabla u, \nabla(\eta \bar{u})>+\int_{\partial M} \eta \bar{Q}<P, n>+\int_{M} \eta\left(|u|^{2}-1\right)|u|^{2} \\
& =-\int_{\eta}|\nabla u|^{2}-\int_{M} \bar{u}<\nabla u, \nabla \eta>+\int_{\partial M} \eta \bar{Q}<p, n> \\
& +\int_{M} \eta\left(|u|^{2}-1\right)|u|^{2} .
\end{aligned}
$$

\section{Local existence and blow-up criterion}

We will apply the Strichartz inequality on a compact manifold to obtain the local existence theory for the equation (1.1). First we recall the Strichartz inequality on compact manifold with boundary or without boundary: the pair $(p, q)(p, q \geq 1)$ is called an admissible if

$$
\frac{2}{p}+\frac{d}{q}=\frac{d}{2},(p, q, d) \neq(2, \infty, 2)
$$

then

$$
\left\|e^{i t \Delta} f\right\|_{L^{p}\left(I, L^{q}(M)\right)} \leq C(I)\|f\|_{H^{\frac{4}{3 p}}(M)}
$$

for any finite interval. Due to the Duhamel principle this inequality implies the inhomogeneous estimate: if $(p, q)$ is an admissible pair as above, then

$$
\left\|\int_{0}^{t} e^{i(t-s) \Delta} f(s) d s\right\|_{L^{p}\left((0, T), L^{q}(M)\right)} \leq C(T)\|f\|_{L^{1}\left((0, T), H^{\frac{4}{3 p}}(M)\right)}
$$

for any finite $T>0$. We referee the reader $[\mathbf{2}]$ for the proof.

The main result of this section is the following local existence and blow up criterion. 
LEMMA 3.1. If $\|\phi\|_{H^{1}(M)} \leq c_{0}$, there exists $T_{0}$ depending only on $c_{0}$ such that there is a unique solution to the equation in the space $C\left(\left[0, T_{0}\right], H^{1}(M)\right) \cap$ $L^{p}\left(\left[0, T_{0}\right], W^{\sigma, q}(M)\right)$ for the admissible pair $(p, q)$ with

$$
\sigma=1-\frac{4}{3 p}>\frac{2}{q}
$$

Moreover, if maximum time $T_{\max }$ is finite, then the $H^{1}$ norm of $u$ blows up.

Proof. By the standard elliptic theory, there exists $\tilde{Q} \in C^{3}$ satisfying

$$
\begin{aligned}
\Delta \tilde{Q} & =f(Q)-i Q_{t} \text { on } \partial M \\
\tilde{Q} & =Q \text { on } \partial M
\end{aligned}
$$

Writing $v=u-\tilde{Q}$, then $v$ satisfies the following equation

$$
\left\{\begin{array}{l}
i v_{t}+\Delta v=h \\
v(0)=\phi-\tilde{Q}(0) \\
\left.v\right|_{\partial M}=0
\end{array}\right.
$$

where $h=f(v+\tilde{Q})-i \tilde{Q}_{t}+\Delta \tilde{Q}$. Notice that by (3.1) and (3.2), $h$ vanishes on $\partial M$. Let $e^{i t \Delta}$ be the linear Schrodinger evolution operator with homogeneous boundary condition. We know that $e^{i t \Delta}$ is a group of unitary operators on $H_{0}^{1}(M)$ to itself. By the Duhamel formula, the equation (3.3) is equivalent to the integral equation

$$
v(t)=e^{i \Delta t} v(0)+i \int_{0}^{t} e^{i \Delta(t-s)} h(s) d s .
$$

We shall prove the local existence result by applying the Strichartz estimate and Banach fixed point theory.

Define:

$$
\begin{array}{r}
B=\left\{u \in C\left(\left[0, T_{0}\right], H^{1}(M)\right) \cap L^{p}\left(\left[0, T_{0}\right], W^{\sigma, q}(M)\right):\right. \\
\left.\|u\|_{L^{\infty}\left(\left[0, T_{0}\right], H^{1}(M)\right)} \leq C,\|u\|_{L^{p}\left[0, T_{0}\right], W^{\sigma, q}(M)} \leq C\right\} .
\end{array}
$$

The Sobolev inequality gives us that $W^{\sigma, q}(M) \hookrightarrow L^{\infty}(M)$ (for the Sobolev imbedding theorem on manifolds, a good referee is [8]). If we equip $B$ with the distance $d(u, v)=\|u-v\|_{L^{\infty}\left(\left[0, T_{0}\right], H^{1}(M)\right)}+\|u-v\|_{L^{p}\left(\left[0, T_{0}\right] W^{\sigma, q}(M)\right)}$, it is obvious that $B$ is a complete distance space.

We define the operator $\mathscr{F}$ on $B$ by

$$
\mathscr{F} u(t)=e^{i \Delta t} v(0)+i \int_{0}^{t} e^{i \Delta(t-s)} h(u(s)) d s .
$$

Then

$$
\|\mathscr{F} u(t)\|_{H^{1}(M)} \leq\|v(0)\|_{H^{1}(M)}+\int_{0}^{T_{0}}\|h(s)\|_{H^{1}(M)} d s .
$$

Since $f(u+\tilde{Q})=\left(|u+\tilde{Q}|^{2}-1\right)(u+\tilde{Q})$, we deduce that

$$
\begin{aligned}
& \|f(u+\tilde{Q})\|_{L^{2}(M)} \\
\leq & \mid u \|_{L^{\infty}(M)}\left(\|u\|_{L^{\infty}(M)}+\|\tilde{Q}\|_{L^{\infty}(M)}+1\right)\left(\|u+\tilde{Q}\|_{L^{2}(M)}+\tilde{C}\right) \\
+ & \|\tilde{Q}\|_{L^{\infty}(M)}\left(\|u\|_{L^{\infty}(M)}+\|\tilde{Q}\|_{L^{\infty}(M)}+1\right)\left(\|u+\tilde{Q}\|_{L^{2}(M)}+\tilde{C}\right) \\
\leq & \tilde{C}\left(\|u\|_{L^{\infty}(M)}^{2}+1\right)\left(\|u\|_{L^{2}(M)}+1\right),
\end{aligned}
$$




$$
\begin{aligned}
& \|\nabla f(u+\tilde{Q})\|_{L^{2}(M)} \leq \\
& \left\|u+\left.\tilde{Q}\right|^{2}-1\right\|_{L^{\infty}(M)}\left(\|\nabla \tilde{Q}\|+\|\nabla u\|_{L^{2}(M)}\right) \\
+ & \|\nabla u\|_{L^{2}(M)}\left(\|u\|_{L^{\infty}(M)}+\|\tilde{Q}\|_{L^{\infty}(M)}+1\right)\left(\|u\|_{L^{\infty}(M)}+\|\tilde{Q}\|_{L^{\infty}(M)}\right) \\
+ & \|\nabla \tilde{Q}\|\left(\|u\|_{L^{\infty}(M)}+\|\tilde{Q}\|_{L^{\infty}(M)}+1\right)\left(\|u\|_{L^{\infty}(M)}+\|\tilde{Q}\|_{L^{\infty}(M)}\right) \\
\leq & \tilde{C}\left(1+\|\nabla u\|_{L^{2}(M)}\right)\left(\|u\|_{L^{\infty}(M)}^{2}+1\right) .
\end{aligned}
$$

Here and after we denote $\tilde{C}$ by the constants. They may be different but not depend on $u$ and $T_{0}$.

Now we get the following estimate for $h$

$$
\|h\|_{H^{1}(M)} \leq \tilde{C}\left\{\left(1+\|u\|_{H^{1}(M)}\right)\left(1+\|u\|_{L^{\infty}(M)}^{2}\right)+1\right\} .
$$

Then from the inequality (3.4) we obtain

$$
\|\mathscr{F} u(t)\|_{H^{1}(M)} \leq\|v(0)\|_{H^{1}(M)}+\tilde{C} \int_{0}^{T_{0}}\left(1+\|u(s)\|_{L^{\infty}(M)}^{2}\right)\left(1+\|u(s)\|_{H^{1}(M)}\right) d s .
$$

By the Sobolev inequality we have

$$
\begin{aligned}
\|\mathscr{F} u\|_{L^{\infty}\left(\left[0, T_{0}\right] H^{1}(M)\right.} & \leq\|v(0)\|_{H^{1}(M)} \\
& +\tilde{C} T_{0}\left(1+\|u\|_{L^{\infty}\left(\left[0, T_{0}\right], H^{1}(M)\right)}\right) \\
& +\tilde{C}\left(1+\|u\|_{L^{\infty}\left(\left[0, T_{0}\right], H^{1}(M)\right)}\right) \int_{0}^{T_{0}}\|u\|_{W^{\sigma, q}(M)} d s .
\end{aligned}
$$

Applying the Holder inequality, we show that

$$
\begin{aligned}
& \|\mathscr{F} u\|_{L^{\infty}\left(\left[0, T_{0}\right] H^{1}(M)\right.} \leq\|v(0)\|_{H^{1}(M)} \\
+ & \tilde{C} T_{0}\left(1+\|u\|_{L^{\infty}\left(\left[0, T_{0}\right], H^{1}(M)\right)}\right) \\
+ & \tilde{C} T_{0}^{\gamma}\left(1+\|u\|_{L^{\infty}\left(\left[0, T_{0}\right], H^{1}(M)\right)}\right)\|u\|_{L^{p}\left(\left[0, T_{0}\right], W^{\sigma, q}(M)\right)}^{2} .
\end{aligned}
$$

Here $\gamma=1-\frac{2}{p}$ and it is between 0 and 1 by the assumption.

Then we have

$$
\begin{aligned}
&\|\mathscr{F} u\|_{L^{\infty}\left(\left[0, T_{0}\right] H^{1}(M)\right.} \leq\|v(0)\|_{H^{1}(M)} \\
&+\quad \tilde{C} T_{0}^{\gamma}\left(1+\|u\|_{L^{\infty}\left(\left[0, T_{0}\right], H^{1}(M)\right)}\right)\left(1+\|u\|_{L^{p}\left(\left[0, T_{0}\right], W^{\sigma, q}(M)\right)}^{2}\right)
\end{aligned}
$$

since we can assume $T_{0} \leq 1$.

Similarly by the Strichartz estimate and the Holder inequality, we get

$$
\begin{aligned}
& \|\mathscr{F} u\|_{L^{p}\left(\left[0, T_{0}\right], W^{\sigma, q}(M)\right)} \leq \\
& \left\|e^{i \Delta t}(1-\Delta)^{\frac{\sigma}{2}} v(0)\right\|_{L^{p}\left(\left[0, T_{0}\right], L^{q}(M)\right)} \\
+ & \left\|\int_{0}^{t} e^{i \Delta(t-s)} h(s) d s\right\|_{L^{p}\left(\left[0, T_{0}\right], L^{q}(M)\right)} \\
\leq & C_{0}\left\|(1-\Delta)^{\frac{\sigma}{2}} v(0)\right\|_{H^{\frac{4}{3} p}(M)}+C_{0}\left\|(1-\Delta)^{\frac{\sigma}{2}} h\right\|_{L^{1}\left(\left[0, T_{0}\right], H^{\frac{4}{3 p}}(M)\right)} \\
\leq & C_{0}\|v(0)\|_{H^{1}(M)}+C_{0}\|h\|_{L^{1}\left(\left[0, T_{0}\right], H^{1}(M)\right)} \\
\leq & C_{0}\|v(0)\|_{H^{1}(M)} \\
+ & \tilde{C} T_{0}^{\gamma}\left(1+\|u\|_{L^{p}\left(\left[0, T_{0}\right], W^{\sigma, q}(M)\right)}^{2}\right)\left(1+\|u\|_{L^{\infty}\left(\left[0, T_{0}\right], H^{1}(M)\right)}\right)
\end{aligned}
$$


where in the third inequality we used the definition of $\sigma$.

For $u, w \in B$, the following inequalities hold:

$$
\begin{array}{ll} 
& \|\mathscr{F} u-\mathscr{F} w\|_{L^{\infty}\left(\left[0, T_{0}\right] H^{1}(M)\right)} \\
\leq \quad & \tilde{C} T_{0}^{\gamma}\left(1+\|u\|_{L^{p}\left(\left[0, T_{0}\right], W^{\sigma, q}(M)\right)}^{2}\right. \\
& \left.+\|w\|_{L^{p}\left(\left[0, T_{0}\right], W^{\sigma, q}(M)\right)}^{2}\right)\|u-w\|_{L^{\infty}\left(\left[0, T_{0}\right], H^{1}(M)\right)},
\end{array}
$$

and

$$
\begin{array}{ll} 
& \|\mathscr{F} u-\mathscr{F} w\|_{L^{p}\left(\left[0, T_{0}\right], W^{\sigma, q}(M)\right)} \\
\leq \quad & \tilde{C} T_{0}^{\gamma}\left(1+\|u\|_{L^{p}\left(\left[0, T_{0}\right], W^{\sigma, q}(M)\right)}^{2}\right. \\
& \left.+\|w\|_{L^{p}\left(\left[0, T_{0}\right], W^{\sigma, q}(M)\right)}^{2}\right)\|u-w\|_{L^{\infty}\left(\left[0, T_{0}\right], H^{1}(M)\right)} .
\end{array}
$$

Using the Strichartz estimate and the above inequalities, we conclude that

$$
\mathscr{F} u \in C\left(\left[0, T_{0}\right], H^{1}(M)\right) \cap L^{p}\left(\left[0, T_{0}\right], W^{\sigma, q}(M)\right)
$$

and

$$
\begin{array}{r}
\|\mathscr{F} u\|_{L^{\infty}\left(\left[0, T_{0}\right] H^{1}(M)\right.} \leq\|v(0)\|_{H^{1}(M)}+\tilde{C} T_{0}^{\gamma}\left(1+C^{2}\right)(1+C), \\
\|\mathscr{F} u\|_{L^{p}\left(\left[0, T_{0}\right], W^{\sigma, q}(M)\right)} \leq C_{0}\|v(0)\|_{H^{1}(M)}+\tilde{C} T_{0}^{\gamma}\left(1+C^{2}\right)(1+C) .
\end{array}
$$

Moreover, we have

$$
d(\mathscr{F} u, \mathscr{F} w) \leq \tilde{C} T_{0}^{\gamma}\left(1+C+C^{2}\right) C d(u, w)
$$

Hence if we choose $C$ to be $\max \left(2\|v(0)\|_{H^{1}(M)}, C_{0}\|v(0)\|_{H^{1}(M)}\right)$ and $T_{0}^{\gamma}$ to be $\frac{1}{2 \tilde{C}\left(1+C+C^{2}\right) C}$, then $\mathscr{F}$ maps $B$ into itself and $d(\mathscr{F} u, \mathscr{F} w) \leq \frac{1}{2} d(u, w)$. Thus $\mathscr{F}$ is a contraction map on $B$. So there is a solution $v$ corresponding (3.3). From the proof we observe that $T_{0}$ depends only on $\|v(0)\|_{H^{1}(M)}$ and then $\|v\|_{H^{1}(M)}$ will blow up if the maximal time $T_{\max }$ is finite. Because $v=u-\tilde{Q}$ and $\tilde{Q}$ has compact support, the blow-up criterion for $u$ is obtained. Since the above contraction argument is standard, we refer the reader to $[\mathbf{3}]$ for similar applications.

\section{Global existence}

From Lemma 3.1 we know if $T_{\max }$ is finite, then $\|u\|_{H^{1}(M)}$ approaches to infinity when $t$ tends to $T_{\max }$. So if we can prove that at any finite time $\|u\|_{H^{1}(M)}$ will not blow up, then we get the global result. All of this is reduced to the following result which is essentially a Gronwall inequality involving $\|u\|_{H^{1}(M)}$.

Lemma 4.1. Fix $T>0$ and assume that $u$ is the solution of equation (1.1) in the space $C\left([0, T), H^{1}\right)$. Then $\|u\|_{H^{1}} \leq C_{T}, \forall 0 \leq t<T$.

Proof. In Lemma (2.1), we have proved the following identities for smooth solutions. First,

$$
\partial_{t} \int_{M}|u|^{2}=2 \operatorname{Im} \int_{\partial M}<P, n>\bar{Q}
$$

Second,

$$
\partial_{t} \int_{M}|\nabla u|^{2}+F(u)=2 \operatorname{Re} \int_{\partial M}<P, n>\bar{Q}_{t}
$$


Third,

$$
\begin{aligned}
& \partial_{t} \int_{M} u<\xi, \nabla \bar{u}>-\int_{\partial M} Q \bar{Q}_{t}+\int_{M} \eta u \bar{u}_{t} \\
= & i \int_{M}<\nabla \xi, \nabla u \otimes \nabla \bar{u}>-i \int_{\partial M}|<P, n>|^{2} \\
- & \frac{i}{2} \int_{M} \eta\left(|u|^{2}-1\right)^{2}+\frac{i}{2} \int_{\partial M}\left(|Q|^{2}-1\right)^{2},
\end{aligned}
$$

Fourth,

$$
\begin{aligned}
& \int_{M} i \eta u_{t} \bar{u}=-\int_{M} \eta|\nabla u|^{2} \\
- & \int_{M} \bar{u}<\nabla u, \nabla \eta> \\
+ & \int_{\partial M} \eta \bar{Q}<P, n>+\int_{M} \eta\left(|u|^{2}-1\right)|u|^{2} .
\end{aligned}
$$

By the standard approximation argument (see, for example [3]), we know that the above identities are available for the solution $u$ in Lemma 3.1. We now take $u$ as the solution in Lemma 3.1 and integrate with respect to $t$ in (4.3)

$$
\begin{aligned}
& \int_{M} u<\xi, \nabla \bar{u}>-\int_{M} \phi<\xi, \nabla \bar{\phi}> \\
- & \int_{0}^{t} \int_{\partial M} Q \bar{Q}_{s} d s+\int_{0}^{t} \int_{M} \eta u \bar{u}_{s} d s \\
= & i \int_{0}^{t} \int_{M}<\nabla \xi, \nabla u \otimes \nabla \bar{u}>-i \int_{0}^{t} \int_{\partial M}|<P, n>|^{2} \\
- & \frac{i}{2} \int_{0}^{t} \int_{M} \eta\left(|u|^{2}-1\right)^{2}+\frac{i}{2} \int_{\partial M}\left(|Q|^{2}-1\right)^{2} .
\end{aligned}
$$

If we set $J(t)=\left.\int_{0}^{t} \int_{\partial M}|<P, n\rangle\right|^{2}$, this implies

$$
\begin{aligned}
J(t) & \leq \int_{M}|u||\xi||\nabla|+\int_{M}|\phi||\xi||\nabla \phi|+\int_{0}^{t} \int_{\partial M}\left|Q \bar{Q}_{s}\right| d s \\
& +\left|\int_{0}^{t} \int_{M} \eta u \bar{u}_{s} d s\right|+c \int_{0}^{t} \int_{M}|\nabla u|^{2}+\frac{1}{2} \int_{\partial M}\left(|Q|^{2}-1\right)^{2} \\
& +c \int_{0}^{t} \int_{M}\left(|u|^{2}-1\right)^{2} .
\end{aligned}
$$

Because of $Q \in C^{3}(\partial M \times(-\infty, \infty))$, the terms involving $\phi$ and $Q$ are bounded. Here and after we denote various constants by $c$ depending only on $Q, T$, but not on $u$.

The identity (4.4) implies

$$
\begin{aligned}
\left|i \int_{M} \eta u_{t} \bar{u}\right| & \leq c \int_{M}|\nabla u|^{2}+\int_{M}|u||\nabla u||\nabla \eta|+\int_{\partial M}|\eta \bar{Q} \|<P, n>| \\
& +c \int_{M}\left(|u|^{2}-1\right)^{2}+c \int_{M}|u|^{2}+c .
\end{aligned}
$$


Combining the inequalities (4.5), (4.6) and using the Holder inequality, we get

$$
\begin{aligned}
J(t) & \leq c \int_{M}|u|^{2}+|\nabla u|^{2}+c_{\varepsilon} \int_{0}^{t} \int_{\partial M}|\eta \bar{Q}|^{2} \\
& +\varepsilon \int_{0}^{t} \int_{\partial M}|\langle P, n\rangle|^{2}+c \int_{0}^{t} \int_{M}\left(|u|^{2}-1\right)^{2} \\
& +c \int_{0}^{t} \int_{M}|u|^{2}+|\nabla u|^{2}+c .
\end{aligned}
$$

Then the following inequality holds

$$
\begin{aligned}
J(t) & \leq c \int_{M}|u|^{2}+|\nabla u|^{2}+c \int_{0}^{t} \int_{M}\left(|u|^{2}-1\right)^{2} \\
& +c \int_{0}^{t} \int_{M}|u|^{2}+|\nabla u|^{2}
\end{aligned}
$$

From (4.1) (4.2), we obtain

and

$$
\int_{M}|u|^{2}-\int_{M}|\phi|^{2}=2 \operatorname{Im} \int_{0}^{t} \int_{\partial M}<P, n>
$$

$$
\begin{aligned}
& \int_{M}|\nabla u|^{2}+f(u)-\int_{M}|\nabla \phi|^{2}+f(Q) \\
= & 2 \operatorname{Re} \int_{0}^{t} \int_{\partial M}<P, n>.
\end{aligned}
$$

Set

$$
\gamma(t)=\int_{M}|u|^{2}+|\nabla u|^{2}+f(u)
$$

By the Holder inequality we have

$$
\gamma(t) \leq c \sqrt{J(t)}+c
$$

and

$$
J(t) \leq c+c \gamma(t)+c \int_{0}^{t} \gamma(s) d s .
$$

The inequalities (4.7), (4.8) and the Holder inequality again show that

$$
J(t) \leq c+\varepsilon J(t)+\int_{0}^{t} J(s) d s
$$

with $0<\varepsilon<1$. By the Gronwall inequality, we obtain

$$
J(t) \leq c
$$

for any $0 \leq t<T$. Then from (4.7), we know

$$
\gamma(t) \leq c
$$

The proof is complete.

Now we can prove the global result.

Proof of Theorem 1.1. We only consider the part $[0,+\infty)$ since the case $(-\infty, 0]$ is similar. If $u$ is not global, that is, $T_{\max }$ is finite, then $\|u\|_{H^{1}(M)}$ tends infinity when $t \rightarrow T_{\max }$. However, this contradicts with the apriori estimate $\|u\|_{H^{1}(M)} \leq C_{T_{\max }}$ 
$\forall 0 \leq t<T_{\max }$. The contradiction shows that $u$ is global. Thus, we have proved theorem 1.1. q.e.d.

\section{References}

[1] C. Bu, Nonlinear Schrodinger equation on the semi-infinite line, Chinese Annals of Math. 21A (2000), 112-23.

[2] Matthew D. Blair, Hart F. Smith, and Christopher D. Sogge, On Strichartz estimates for Schrodinger operators in compact manifold with boundary. to appear.

[3] Thierry Cazenave, An Introduction to Nonlinear Schrodinger Equations, Courant Lect. Notes Math. 10, Courant Inst. Math. Sci., New York.

[4] R. Carroll and C. Bu, Solution of the forced nonlinear Schrodinger equation (NLS) using PDE techniques. Appl. Anal. 41 (1991), 3351.

[5] P.Gerard, The Cauchy problem for the Gross-Pitaevskii equation. Ann.I.H.Ponicare-AN, 23(2006) 765-779.

[6] Hongjun Gao, Charles Bu, Neumann inhomogeneous boundary value problem for the $n+1$ complex Ginzburg-Landau equation. Applied Mathematics and Computation, 188(1): 394-398 (2007)

[7] Philippe Gravejat, Decay for travelling waves in the Gross-Pitaevskii equation. Ann. I. H. Poincare,AN 21 (2004) 591C637.

[8] E.Hebey, Nonlinear analysis on manifolds: Sobolev spaces and inequalities, CIMS Lecture Notes, Courant Institute of Mathematical Sciences, Volume 5, 1999

[9] Walter Strauss Charles Bu,An Inhomogeneous Boundary Value Problem for Nonlinear Schrodinger Equation. Journal of Differential Euations, 173, 79-91 (2001).

[10] Y.Tsutsumi, global solutions of the nonlinear Schröinger equations in exterior domains, Commun. Partial Differ. Equ., 8(1983)1337-1374.

[11] Y.Tsutsumi, On global solutions to the initial-boundary value problem for the nonlinear Schröinger equations in exterior demains,Commun.Partial Differ. Equ., 16(1991)885-907.

Department of mathematical sciences, Tsinghua university, Beijing 100084, China E-mail address: nuslma@gmail.com

Department of mathematical sciences, Tsinghua university, Beijing 100084, China 